This item was submitted to Loughborough's Research Repository by the author.

Items in Figshare are protected by copyright, with all rights reserved, unless otherwise indicated.

\title{
Contourlet based multi-exposure image fusion with compensation for multi- dimensional camera shake
}

PLEASE CITE THE PUBLISHED VERSION

http://dx.doi.org/10.5220/0003836001820185

\section{PUBLISHER}

(c) SCITEPRESS

\section{VERSION}

AM (Accepted Manuscript)

\section{PUBLISHER STATEMENT}

This work is made available according to the conditions of the Creative Commons Attribution-NonCommercialNoDerivatives 4.0 International (CC BY-NC-ND 4.0) licence. Full details of this licence are available at: https://creativecommons.org/licenses/by-nc-nd/4.0/

\section{LICENCE}

CC BY-NC-ND 4.0

\section{REPOSITORY RECORD}

Saravi, Sara, and Eran A. Edirisinghe. 2019. "Contourlet Based Multi-exposure Image Fusion with Compensation for Multi-dimensional Camera Shake”. figshare. https://hdl.handle.net/2134/21856. 


\title{
CONTOURLET BASED MULTI-EXPOSURE IMAGE FUSION WITH COMPENSATION FOR MULTI-DIMENSIONAL CAMERA SHAKE
}

\author{
S.Saravi, E.A.Edirisinghe \\ Digital Imaging Group, Loughborough University, Loughborough, United Kingdom \\ S.Saravi@lboro.ac.uk,E.A.Edirisinghe@lboro.ac.uk
}

Keywords: Image registration, Multi-exposure Image Fusion, Translational and Rotational Camera Shake, Wavelet Based Contourlet Transform, CPD, SIFT, RANSAC

\begin{abstract}
Multi-exposure image fusion algorithms are used for enhancing the perceptual quality of an image captured by sensors of limited dynamic range by rendering multiple images captured at different exposure settings. One practical problem overlooked by existing algorithms is the compensation required for image deregistration due to possible multi-dimensional camera shake that results within the time gap of capturing the multiple exposure images. In our approach RANdom SAmple Consensus (RANSAC) algorithm is used to identify inliers of key-points identified by the Scale Invariant Feature Transform (SIFT) approach subsequently to the use of Coherent Point Drift (CPD) algorithm to register the images based on the selected set of key points. We provide experimental results on set of images with multi-dimensional (translational and rotational) to prove the proposed algorithm's capability to register and fuse multiple exposure images taken in the presence of camera shake providing subjectively enhanced output images.
\end{abstract}

\section{INTRODUCTION}

In the past decade there have been significant developments in the field of High Dynamic Range (HDR) imaging technology. However, the lack of advances in image/video encoding algorithms and display technology makes it important to find alternatives to rendering HDR scenes using SDR imagery. Multi-exposure image fusion involves the fusion of multiple consecutive images of the same scene taken at quick succession by a SDR camera. A practical problem that arises is camera shake that can cause severe de-registration of the multiple images that invalidates the direct applicability of many existing algorithms. To this effect we propose the use of an image registration algorithm as a preprocessing stage to multi-exposure image fusion.

A significant number of multi-exposure image fusion algorithms have been proposed in the relevant literature [1-2]. However, only few algorithms focus on the problem of camera shake [3-4].

Image fusion can take place on pixel-level, feature-level, and decision level. In the literature, image fusion has been based on pyramidal fusion, contourlet fusion and wavelet fusion. The wavelet transform (WT) results are acceptable in natural images, but smooth edges cannot be detected powerfully because it is restricted to three directions, horizontal, vertical and diagonal. Contourlet transform (CT) is a two dimensional transform which has the capability to effectively represent images containing curves and features. In CT, multiscale and multi-direction analyses are done separately, using the Laplacian Pyramid (LP) transform and then the Directional Filter Bank (DFB). This method is redundant because of the LP transform and the fact that multi-resolution structures are not constant since the number of directions in DFB are variable. WBCT [5] is a solution for above weakness which is non-redundant and has a multi-resolution structure. The advantage of using WBCT is that it solves the problems of multi-scale localization, directionality \& anisotropy.

In this paper we present a WBCT approach that uses a number of fusion rules for multi-exposure image fusion and compensates for camera shake. Section 2 presents the proposed system in details. Experimental results are provided in section 3 and finally section 4 concludes the proposed work.

\section{PROPOSED SYSTEM}

The proposed system consists of two key modules. The first module uses a selected base image of a multi exposure image set to register all remaining images. The second module fuses the registered, multi-exposure image set. 


\subsection{Image-Registration}

The proposed approach is based on the selection of a significant set of matching points, i.e. key-points, between a selected base image and an image to be registered and subsequently using them to calculate the transformation matrix for image registration.

\subsubsection{SIFT based key-point selection}

SIFT [6] is an algorithm that is capable of detecting and describing local features of an image. It's invariance to rotation, scale and translation has made it a popular algorithm in many areas of computer vision. SIFT is also invariant to illumination changes and robust to local geometric distortion.

In the proposed approach to image registration we select a base image from amongst the set of multiexposure images and an image to be registered to it and use SIFT to find significant key-points in both images. Due to large number of feature points that may be selected by SIFT in execution the matching of key-points between two images to be registered; it is likely that two non-corresponding points may match as they result in the minimum distance. Therefore reducing keypoint outliers prior to the matching key-points will improve the reliability of matching and hence the outcome of the final task.

\subsubsection{Using RANSAC to remove matching point outliers}

RANSAC [7] algorithm is an iterative method to approximate factors of a mathematical form, from a set of experimental data which include outliers. RANSAC is able to do robust estimations of the model factors; it can estimate the parameters with a high degree of accuracy even when a significant amount of outliers are present in the data set.

In our approach all SIFT key-points points resulting from the stage described in section 2.1.1 from the base image and the image being registered are fed to RANSAC algorithm. It fits a model to these inlier points and tests the points from the image being registered against the fitted model, and if a point fits to the model it will be regarded as inlier. The model is recalculated from all inliers and then the error is estimated relative to the model. The outlier key-points are finally removed from the keypoints of the image.

\subsubsection{CPD algorithm for registration}

In this section we describe the use of the CPD [8] algorithm to register the images, accordingly preparing them to be fused in the subsequent stage of the proposed approach. CPD is based on 'Point Set Registration' and aims to form links between two given sets of points to find the corresponding features and the necessary transformation of these features that will allow the images to be registered.

There are two methods for registering an image in $\mathrm{CPD}$, rigid and non-rigid point set approaches, based on the transformation model principal. The key characteristic of a rigid transformation is “distance between points are preserved", which means it just can be used in the presence of translation, rotation, and scaling, but not under scaling and skew. Affine, the transformation we have used in our work is a non rigid transformation, which provides the opportunity of registering under non-uniform scaling and skew.

\subsection{Multi-Exposure Image Fusion}

Once all images are registered to the base image we use WBCT to identify regions of maximum energy from within the multiple exposure images, with the idea of combining these to form the perceptually best quality fused image.

\subsubsection{Multi-exposure image fusion}

The basic idea of the fusion algorithm is to compare the corresponding sub-bands of the WBCT decomposition of each multi-exposure image set and to determine the one with the highest energy, i.e. most detailed. We propose the use of different fusion rules depending on the frequency band of each subband being fused, as follows:

Fusion of High Frequency Contourlet Sub-bands: The high frequency sub-bands contain details of an image such as texture and edges where as the low frequency sub-bands contains details of more spread-out nature or fuzzy, such as background information. By calculating the absolute energy of high-frequency coefficients, the energy of a region can be obtained. A higher value means sharper changes. Region energy $E$ of a high frequency subband $\mathrm{E}_{\mathrm{H}}$ (where $\mathrm{H}=(\mathrm{l}, \mathrm{m}, \mathrm{n})$, l-level of wavelet decomposition, m-LH, HL and HH bands of wavelet decomposition, n-directional Contourlet sub-band) of an image $\mathrm{X}$ can be obtained as follows:

$$
\mathrm{E}_{\mathrm{H}}^{(\mathrm{X})}=\sum_{(\mathrm{x}, \mathrm{y}) \in \mathrm{H}} \mathrm{f}_{\mathrm{H}}^{(\mathrm{X})}(\mathrm{x}, \mathrm{y})^{2}
$$

$f_{H}(x, y)$ is the coefficient at location $(x, y)$ of the high frequency sub-band $\mathrm{H}=(\mathrm{l}, \mathrm{m}, \mathrm{n})$.

Considering that the sub-band of the multiexposure image having highest detail will have the 
highest absolute energy, the $\mathrm{H}$ sub-band that contributes towards the fused image's $\mathrm{H}$ sub-band can be defined as:

$$
f_{H}^{(F)}(i, j)=f_{H}^{(Y)}, E_{H}^{(Y)}=\left.\max \left(E_{H}^{(i)}\right)\right|_{i=1,2, \ldots n}
$$

Where $\mathrm{n}$ is the number of exposures considered.

Fusion of Low Frequency Contourlet Sub-bands: The Low frequency sub-bands contain the fuzzy, spread-out information. Thus the fusion rule adopted is based on the region variances. The idea is to divide each low frequency sub-band to $i \times j$ rectangular sub regions and calculate the variance of each sub region which can be obtained as follows:

$$
\mathrm{V}_{\mathrm{L}}^{(\mathrm{X})}(\mathrm{x}, \mathrm{y})=\sum_{(\mathrm{x}, \mathrm{y}) \in(\mathrm{i} \times \mathrm{j})}\left(\mathrm{f}_{\mathrm{L}}^{(\mathrm{x})}(\mathrm{i}, \mathrm{j})(\mathrm{x}, \mathrm{y})-\overline{\mathrm{f}}_{\mathrm{L}}^{(\mathrm{X})}(\mathrm{i}, \mathrm{j})\right)^{2}
$$

is the mean of all the coefficients in the rectangular sub region $i \times j$. Higher result in variance corresponds to more details. Fusion of the low frequent sub-bands can be obtained from equation below:

$$
\mathrm{f}_{\mathrm{L}}^{(\mathrm{F})}(\mathrm{i}, \mathrm{j})=\mathrm{f}_{\mathrm{L}}{ }^{(\mathrm{Y})}, \mathrm{E}_{\mathrm{L}}{ }^{(\mathrm{Y})}=\left.\max \left(\mathrm{E}_{\mathrm{L}}{ }^{(\mathrm{i})}\right)\right|_{\mathrm{i}=1,2, \ldots \mathrm{n}}
$$

Where $\mathrm{n}$ is the number of exposures considered. Fusion of Low Frequency Wavelet Sub-bands: The low-pass wavelet sub-band of fused image is calculated by averaging the low pass wavelet subbands of the multiple exposure images, as follows:

$$
A_{\mathrm{L}}^{(\mathrm{F})}(\mathrm{i}, \mathrm{j})=\frac{1}{n} \sum_{\mathrm{a}=1}^{\mathrm{n}} \mathrm{f}_{\mathrm{L}}^{(\mathrm{a})}(\mathrm{i}, \mathrm{j}),(\mathrm{i}, \mathrm{j}) \in \mathrm{LL}
$$

Where $n$ is the number of the multi exposure images and $f(i, j)$ is a coefficient from the low pass sub-band of the wavelet transform.

\subsection{Reconstructing the Fused Image}

After obtaining low and high frequency sub-bands of $\mathrm{CT}$ and low frequency sub-band of WT as above, the fused image is constructed using inverse WBCT.

\section{EXPERIMENTAL RESULTS}

Experiments were conducted five sets of multiexposure images obtained with a hand held camera (i.e. to add camera shake) for testing the image registration prior to the multi-exposure image fusion. The sample results illustrated in figure 1 for one image set prove that the proposed approach is capable of producing fused images of noticeably good quality.

The images were taken allowing the free movement of the camera, i.e. allowing shake. All images were Gamma corrected before processing by the proposed algorithms. Figure 1(a) is the base image (over exposed) and Figure 1(b) is an unregistered image of a different exposure setting (under exposed), which is intended to be aligned with the base image. The SIFT key feature points found as a result of applying SIFT on the underexposed image is illustrated in Figure 1(c) [note the SIFT key-points of the underexposed image is not illustrated but could have been illustrated similarly]. Subsequently using the RANSAC algorithm (Figure 1(d)) the mismatched points are eliminated and finally by using the CPD algorithm the two images are registered [observe key-point matching with [Figure 1(f)) and without (Figure 1(e)) prior registration]. Figure $1(\mathrm{~g})$ illustrates the fusion result without prior registration of images indicating a blurry and smudgy nature on some parts of the image while Figure 1(h) illustrates the positive impact of prior image registration using the proposed algorithm. It can be seen that the quality has been increased in the form of increased sharpness and more details being observable.

\section{CONCLUSIONS}

In this paper a multi-exposure fusion approach has been proposed that provides effective compensation for camera shake. We have provided experimental results on a standard set of multi-exposure images with movement and a specifically captured additional set of images to analyse the performance of the proposed approach. Results indicate that the proposed approach is capable of effective multiexposure image fusion under camera shake.

\section{REFERENCES}

[1] Zafar, I., Edirisinghe, E., Bez, H., 2006. Multiexposure \& focus image fusion in transform domain, IET Intl. Conf. on Visual Info. Eng. IEE.

[2] Alsam, A., 2010. Multi Exposure Image Fusion. Norsk informatik konferanse. Akademisk Forlag.

[3] Tomaszewska, A., Mantiuk, R., 2007. Image registration for multi-exposure high dynamic range image acquisition. Intl. Conf. on Comp. Graphics, Visualization \& Vision. Springer.

[4] Lee, S., Wey, H., 2009. Image registration for multi-exposed HDRI and motion Deblurring. The Intl. Society for Optical Eng. Proc. of SPIE 
[5] Eslami, R., Radha, H., 2005. Wavelet-based Contourlet Packet Image Coding. Conf. on Info. Sciences and Systems. CISS.

[6] Lowe, D., 2004. Distinctive image features from scale invariant key-points. Intl. Comp. Vision.

[7] Fischler, M. A., Bolles, R. C., 1981. Random sample consensus: A paradigm for model fitting with applications to image analysis and automated cartography. ACM Communications.

[8] Myronenko A., 2010. Point-Set Registration: Coherent Point Drift. IEEE Trans. on Pattern Analysis and Machine Intelligence, IEEE.

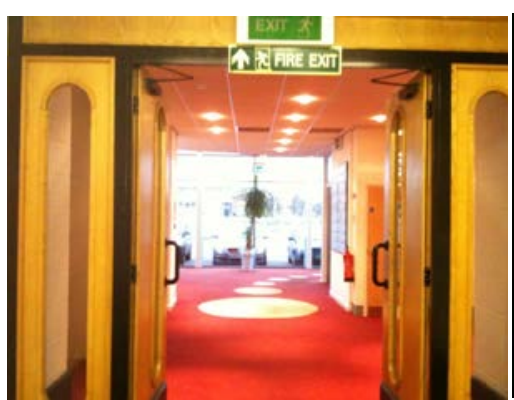

(a) Over Exposed

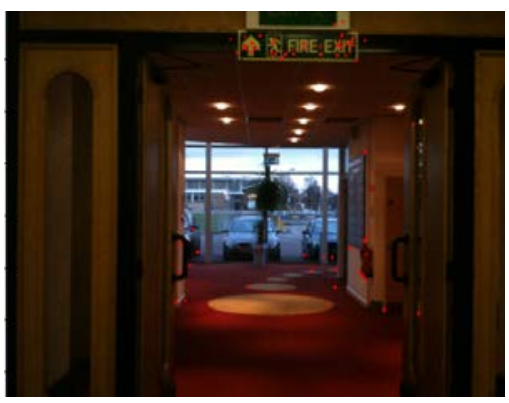

(d) Keypoints that remain after RANSAC

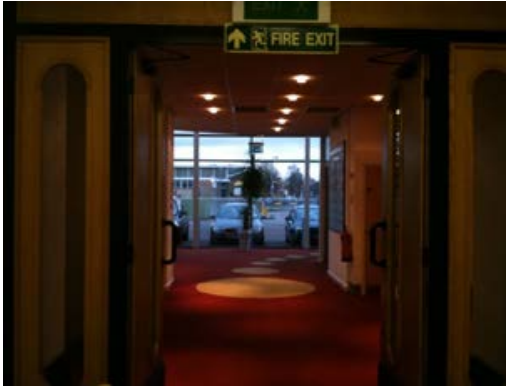

(b) Under Exposed

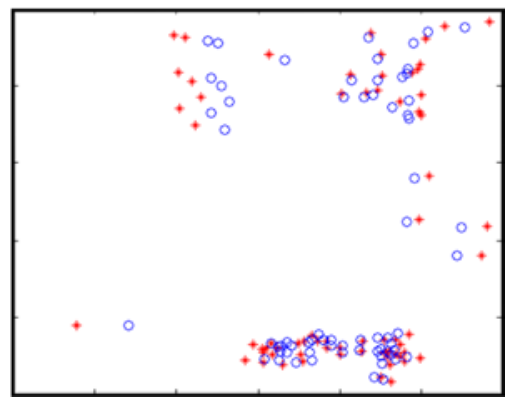

(e) Keypoints before registration using CPD

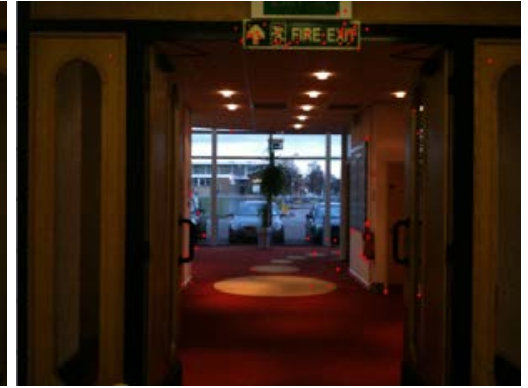

(c) SIFT keypoint extraction on under exposed image

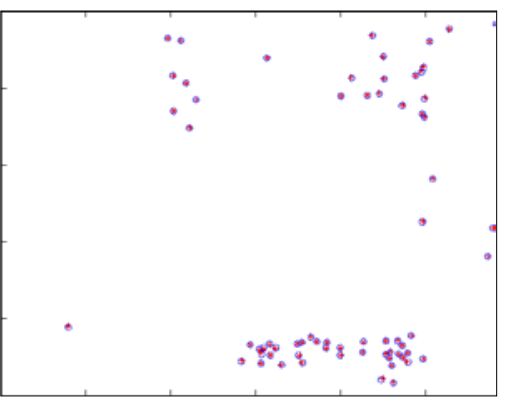

(f) Keypoints after registration using CPD

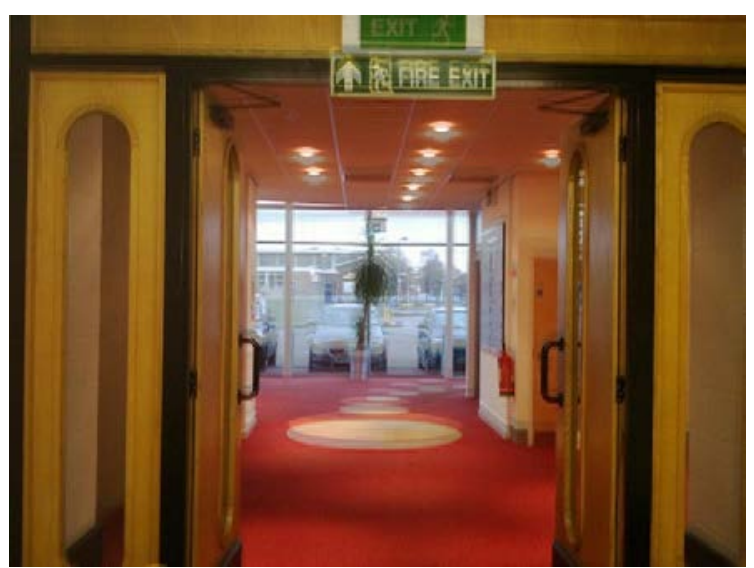

(g) Fused image without prior registration

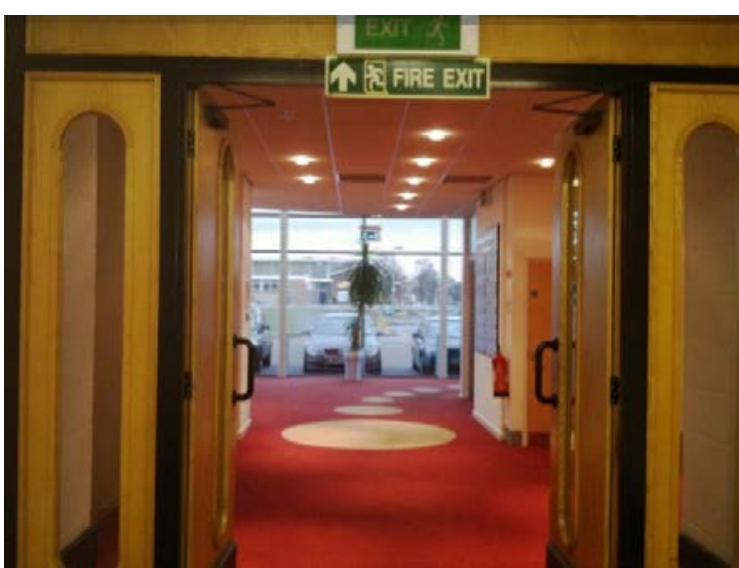

(h) Fused image with prior registration

Figure 1: Experimental dataset 